\title{
Establishing a Prediction Model for Coronary Angiography Based on Coronary Risk Factors
}

\author{
Tsochiang MA, ${ }^{1}$ PhD, Gwo-Ping Jong,,${ }^{2,3}$ MD, Kuo-Chiang UENG, ${ }^{4}$ MD, \\ and Pesus CHOU, ${ }^{2}$ DrPH
}

\begin{abstract}
SUMMARY
The aim of the present study was to establish an evidence-based effective prediction model for improving the accuracy and priority for undertaking coronary angiography.

The sample population consisted of 2002 coronary angiography patients. Our data were taken from claim forms provided by the Taiwanese Bureau of National Health Insurance. The results were tested using chi-square automatic interaction detection to establish a prediction model using coronary risk factors.

We found significant variation across homogeneous groups, with the probabilities of developing coronary heart disease (CHD) varying according to risk factors such as sex, hypertension, diabetes, age, and physical inactivity. The study also explored the influence of interactions among patient characteristics. The sensitivity, specificity, and positive predictive value of our study were $92.0 \%, 35.4 \%$, and $76.5 \%$ respectively, indicating the diagnostic accuracy of the model is at least as high as the treadmill exercise test.

The results suggest that the accuracy of a decision concerning the performance of cardiac angiography can be significantly enhanced by an evidence-based effective prediction model that takes interactions between risk factors into account. This model also helps to priortize patients waiting to undergo coronary angiography. (Int Heart J 2005; 46: 57-68)
\end{abstract}

Key words: Prediction model, Risk factors, Coronary heart disease, Coronary angiography, Chi-square automatic interaction detection

CORONARY angiography is used to evaluate suspected coronary heart disease (CHD) patients. Unlike the treadmill exercise electrocardiography (ECG) test, it is an invasive diagnostic technique, making it a procedure that is not entirely safe. According to the results of previous research, several significant vascular complications occur in approximately $1 \%$ of patients, including hemorrhaging, distal embolization, false aneurysm, and even death. ${ }^{1,2)}$ Inappropriate use of this technique is therefore a serious concern.

From the ${ }^{1}$ Department of Health Service Management, China Medical University, Taichung, ${ }^{2}$ Community Medical Research Center and Institute of Public Health, National Yang-Ming University, Taipei, and ${ }^{3}$ Division of Cardiology, Armed Force Taichung General Hospital, ${ }^{4}$ Division of Cardiology, Shung-Shan Medical University Hospital, Taichung, Taiwan, ROC.

Address for correspondence: Gwo-Ping Jong, MD, Division of Cardiology, Armed Force Taichung General Hospital, No 348, Chung-Shan Rd, Sect 2, Taiping, 411, Taichung, Taiwan, ROC.

Received for publication February 3, 2004.

Revised and accepted August 30, 2004. 
Currently, the decision to conduct coronary angiography is made according to the treadmill exercise ECG test results and nuclear myocardial perfusion scanning, however, the present normal or near-normal rate of coronary angiography, which ranges from 20 to $24 \%$, clearly requires improvement. ${ }^{3,4)}$ This necessitates the establishment of an evidence-based effective prediction model.

The first step is the assessment of the risk factors of CHD, including variables such as hypertension, sex, age, lipid profile, physical inactivity, smoking, diabetes, family history of cardiovascular disease, and obesity. These variables have been identified by numerous studies, ${ }^{5-15)}$ however, because of their reliance on regression models, they are limited in their application to current studies. There are two main reasons for this.

1. Variables developed from the CHD database are generally classified as categorical data; using regression models for the identification of the predictors of outcomes for CHD is therefore less than ideal.

2. Usually, regression models assume that the effects of the independent variables are linear and additive. This assumption obviously does not hold true in the research of CHD, where interactions among categorical variables are common. For example, the interaction between sex and age for CHD patients has been revealed in a previous study. ${ }^{16)}$

Although we used the risk factors from previous studies as independent variables, we moved beyond those studies by ensuring that interactions were taken into account. By using a tree diagram to estimate the chances of developing cardiovascular disease for each homogenous group, it is hoped the accuracy of decisions concerning coronary angiography can be increased and its inappropriate use decreased.

\section{Methods}

Study design: This study is a prospective study. The data for our analysis came from the coronary angiography claim forms provided by the National Health Insurance Bureau in Taichung, Taiwan, submitted between January and December of 2002. The data include hospital name, sex, date of birth, patient ID, ID number, insurance ID, ICD9 code, clinical diagnosis, reason for coronary angiography, cholesterol level, smoking history, family history of cardiovascular disease, level of physical activity, doctor ID, and date. The presence of diabetes, obesity, and/or hypertension was also recorded on the form. The medical record can be retrieved if there are any data missing from the form. The inclusion criteria were: (1) clinical evidence of angina pectoris, and (2) having tested positive in a treadmill exercise test or nuclear myocardial perfusion scan. Data were excluded if (1) missing data could not be obtained from medical records, or (2) if any of the 
following were present on the claim form: acute myocardial infarction, history of myocardial infarction, or an electrocardiogram showing a possible old myocardial infarction or unstable angina pectoris. A total of 2024 samples were collected, with 22 subsequently being excluded. The analytic dataset therefore was 2002 suspected CHD patients.

Definition of variables: The outcome variable will be dichotomized into "insignificant" and "significant" depending on the results of coronary angiography (at least one vessel showing more than $50 \%$ stenosis according to quantitative coronary angiography (QCA) is defined as "significant"); any result other than significant will be assigned to the "insignificant" category.

Independent variables: In this study, the characteristics of suspected patients included as independent variables were chosen according to the following rules: they must (1) appear general enough to include the wide range of patient factors of interest, (2) show no overlap, and (3) have at least some theoretical basis. The measures used to operationalize the variables are listed in Table I.

Statistical analysis: Univariate statistics were used to describe the distribution in age, sex, obesity, smoking, family history, hypertension, cholesterol level, level of physical activity, and diabetes.

Exhaustive chi-square automatic interaction detection (CHAID) was utilized to identify the risk factors of $\mathrm{CHD}$ for use in establishing the prediction model. CHAID, developed by Kass ${ }^{17)}$ is a highly efficient, multivariate, statistical technique for segmentation or tree growing. Used as a criterion for the signif-

Table I. Definition of Variables

\begin{tabular}{|c|c|c|}
\hline Variable & Type & Operation definition \\
\hline \multicolumn{3}{|l|}{ Dependent variable } \\
\hline $\begin{array}{l}\text { Outcomes of coronary } \\
\text { angiography }\end{array}$ & Category & $\begin{array}{l}\text { 0: Insignificant; } 1 \text { : significant } \\
\text { (At least one vessel stenosis more than } 50 \% \text { by QCA) }\end{array}$ \\
\hline \multicolumn{3}{|l|}{ Independent variable } \\
\hline Sex & Category & 1: male; 2 female \\
\hline Age & Continuous & Current date-birthday \\
\hline Obesity & Category & 0 : no $($ BMI $<25) ; 1:$ yes $(25 \leqq$ BMI $<30) ; 2$ : yes $($ BMI $\geqq 30)$ \\
\hline Smoking & Category & $\begin{array}{l}0 \text { : no smoking } \\
1 \text { : current smoking }\end{array}$ \\
\hline $\begin{array}{l}\text { Family history of } \\
\text { cardiovascular disease }\end{array}$ & Category & 0: no; 1 : yes \\
\hline Hypertension & Category & $\begin{array}{l}0 \text { : no hypertension, blood pressure (BP) normal; 1: Mild hyper- } \\
\text { tension, } 140 / 90<\mathrm{BP}<159 / 99 ; 2 \text { : Moderate hypertension, 160/ } \\
100<\mathrm{BP}<179 / 109 ; 3 \text { : Severe hypertension, BP }>180 / 110 \text {. }\end{array}$ \\
\hline Hyperlipidemia & Category & $\begin{array}{l}0 \text { : No hyperlipidemia; } 1 \text { : Triglyceride } \geqq 200 \mathrm{mg} / \mathrm{dL} \text { or total cho- } \\
\text { lesterol } \geqq 200 \mathrm{mg} / \mathrm{dL}\end{array}$ \\
\hline Physical inactivity & Category & 0 : no (at least 30 minutes exercise third a week); 1 : yes \\
\hline Diabetes & Category & $0:$ no; 1 : yes \\
\hline
\end{tabular}

$\mathrm{BMI}=$ body mass index. 
icance of a statistical test, CHAID evaluates all the values of a potential predictor variable. While it merges those values judged to be statistically homogeneous (similar) with respect to the dependent variables, it maintains all other values that are heterogeneous (dissimilar). It then selects the best predictor variable to form the first branch in the decision tree, so that each node is made up of groups of homogeneous values of the selected variable. This process continues repeatedly until the tree is complete. With CHAID, the chi-square test is used to determine significance when the dependent variable is categorical, and the F test is used when it is continuous.

Exhaustive CHAID, a modification developed by Biggs, et $a l^{18)}$ was designed to address some of the weaknesses of the CHAID method. In particular, CHAID occasionally fails to find the optimal split for a variable, since it ceases to merge categories as soon as it discovers that all those remaining are statistically different. Exhaustive CHAID remedies this by continuing to merge categories of the predictor variable until only two super categories remain. Examining the series of mergers for the predictor yields the set of categories that gives the strongest association with the target variable, and computes an adjusted $P$ value for that association. Exhaustive CHAID can therefore obtain the best split for each predictor, and then choose which predictor to split on by comparing the adjusted $P$ value. This makes exhaustive CHAID an efficient statistical method for eliminating the interactions between variables, which undermine the results of current studies.

CHAID has already been successfully implemented in several previous studies. Huang, et al, for example, employed it to identify the characteristics of people who were vulnerable to mental problems. ${ }^{19)}$ This study proves that CHAID can effectively reduce the complexities of large datasets to much simpler sets of relationships between handfuls of important predictors. ${ }^{20)}$

Finally, the sensitivity, specificity, and positive predicted value were calculated to compare with both the treadmill exercise ECG test and the nuclear myocardial perfusion scan values according to a misclassification matrix table. The misclassification matrix could give the estimated risk of misclassification made using the tree. Each case in the cell of the table was classified into one of the two categories according to a predetermined rule. If the percentage appearing at the end-level node of the tree is above $50 \%$, then all the cases in that node will be predicted as having coronary artery disease (CAD). Otherwise, these patients will be classified as without CAD. Take node 1 of the tree (Figure) as an example. As can be seen, if the percentage is above $50 \%$, then all 174 cases in this node are predicted as having CAD. However, only $95 \%$ of the cases (166) are diagnosed as having $\mathrm{CAD}$ while the rest 5\% (8) are without CAD. This implies that 8 cases are misclassified (false positive). Similarly, the percentage shown in node 5 of the 
tree is below $50 \%$. All 46 cases in this node are hence predicted to be without CAD. Nevertheless, $13 \%$ of the cases (6) are diagnosed as having CAD, meaning that they are wrongly classified (false negative). In addition, the misclassification matrix shows exactly what types of errors are being made. The diagonal elements (upper left and lower right) of the table represent correct classifications, while the off-diagonal elements (lower left and upper right) represent misclassifications. The software used in this study is SPSS Answer Tree 3.0.

$$
\begin{aligned}
& \text { Sensitivity }(\%)=\frac{\text { True-positive }}{\text { True-positive }+ \text { false-negative }} \times 100 \% \\
& \text { Specificity }(\%)=\frac{\text { True-negative }}{\text { True-negative }+ \text { false-positive }} \times 100 \% \\
& \text { Positive predictive value }(\%)=\frac{\text { True-positive }}{\text { True-positive }+ \text { false-positive }} \times 100 \%
\end{aligned}
$$

\section{Results}

Description statistics: Out of the 2002 eligible samples, 1392 (69.5\%) obtained a significant coronary angiography result, the rest being insignificant. The age ranged from 19 to 93 , with a mean age of 65 , and over half $(61.4 \%)$ were males. Only approximately $9 \%$ of the patients (177) had a family history of CAD, and $28 \%$ suffered from diabetes. Using body mass index (BMI) to measure obesity, we found $1210(60.4 \%)$ patients had a normal BMI (less than 25), 649 (32.4\%) had a BMI between 25 and 30, and 143 (7.1\%) patients had a BMI above 30. Over half of the patients $(51.2 \%)$ reported exercising for at least 30 minutes, three times a week. The distributions of smoking, hypertension, and cholesterol are

\begin{tabular}{|c|c|c|c|c|c|}
\hline \multirow{2}{*}{ Characteristic } & \multicolumn{2}{|c|}{ Significant CAD $(n=1392)$} & \multicolumn{2}{|c|}{ Insignificant CAD $(n=610)$} & \multirow{2}{*}{$\begin{array}{c}\text { Total } \\
(n=2002)\end{array}$} \\
\hline & Male & Female & Male & Female & \\
\hline Number of results (\%) & $943(47.1)$ & $449(22.4)$ & $286(14.3)$ & $324(16.2)$ & $2002(100)$ \\
\hline Hypertension, mild & $234(11.7)$ & $95(4.7)$ & $57(2.8)$ & $54(2.7)$ & $440(22.0)$ \\
\hline Hypertension, moderate & $206(10.3)$ & $99(4.9)$ & $42(2.1)$ & $58(2.9)$ & $405(20.2)$ \\
\hline Hypertension, severe & $136(6.8)$ & $131(6.5)$ & $27(1.3)$ & $86(4.3)$ & $380(19.0)$ \\
\hline Current smoking (\%) & $537(26.8)$ & $18(0.9)$ & $160(8.0)$ & $20(1.0)$ & $735(36.7)$ \\
\hline Diabetes $(\%)$ & $296(14.8)$ & $169(8.4)$ & $42(2.1)$ & $56(2.8)$ & $563(28.1)$ \\
\hline Hyperlipidemia (\%) & $367(18.3)$ & $106(5.3)$ & $47(2.3)$ & $80(4.0)$ & $600(30.0)$ \\
\hline $\mathrm{BMI}<25$ & 499 (24.9) & $356(17.8)$ & $178(8.9)$ & $177(8.8)$ & $1210(60.4)$ \\
\hline $\mathrm{BMI} \geqq 25$ and $<30$ & $289(14.4)$ & $156(7.8)$ & $88(4.4)$ & $116(5.8)$ & $649(32.4)$ \\
\hline $\mathrm{BMI} \geqq 30$ & $52(2.6)$ & $41(2.0)$ & $19(1.0)$ & $31(1.5)$ & $143(7.1)$ \\
\hline Physical inactivity (\%) & $484(24.2)$ & $272(13.6)$ & $99(5.0)$ & $121(6.0)$ & $976(48.8)$ \\
\hline Family history of CAD (\%) & $89(4.4)$ & $42(2.1)$ & $22(1.1)$ & $24(1.2)$ & $177(8.8)$ \\
\hline
\end{tabular}
shown in Table II.

Table II. Baseline Characteristics of the Study Population

$\mathrm{CAD}=$ coronary artery disease BMI = body mass index. 
CHAID results: We used 0.05 as the level of significance. The results of the initial recategorization of the variables to best predict significant CHD are presented in the Figure. The tree diagram from CHAID shows that the probabilities of developing significant CHD for suspected patients ranged from as high as $95.4 \%$ to as low as $13.0 \%$. Of the 2002 suspected patients, $69.5 \%$ were significant and $30.5 \%$ were insignificant. Gender, hypertension, diabetes, physical inactivity, and age were chosen as predictors of developing significant CHD, with the strongest of these predictors being male gender. This choice was made according to the probability of developing significant CHD being $76.7 \%$ for males and $58.1 \%$ for females, demonstrating the increased vulnerability of males to significant CHD. Further segmentation revealed that, within the male group, hypertension was the most important factor followed by diabetes. Those with hypertension had an $82.1 \%$ probability of developing significant CHD, compared with $63.0 \%$ for those without. Patients with hypertension had an increased probability of developing significant CHD ranging from $78.4 \%$ without diabetes to $90.4 \%$ with diabetes. Physical inactivity also played an important role in males with hypertension, but only affected those with diabetes. Physically inactive male patients with suspected hypertension and diabetes had the highest probability of developing significant CHD (95.4\%), as compared with a lower $81.4 \%$ for physically active patients.

Focusing on the female group, we found age to be the next most significant variable, with the chances of developing significant CHD varying according to

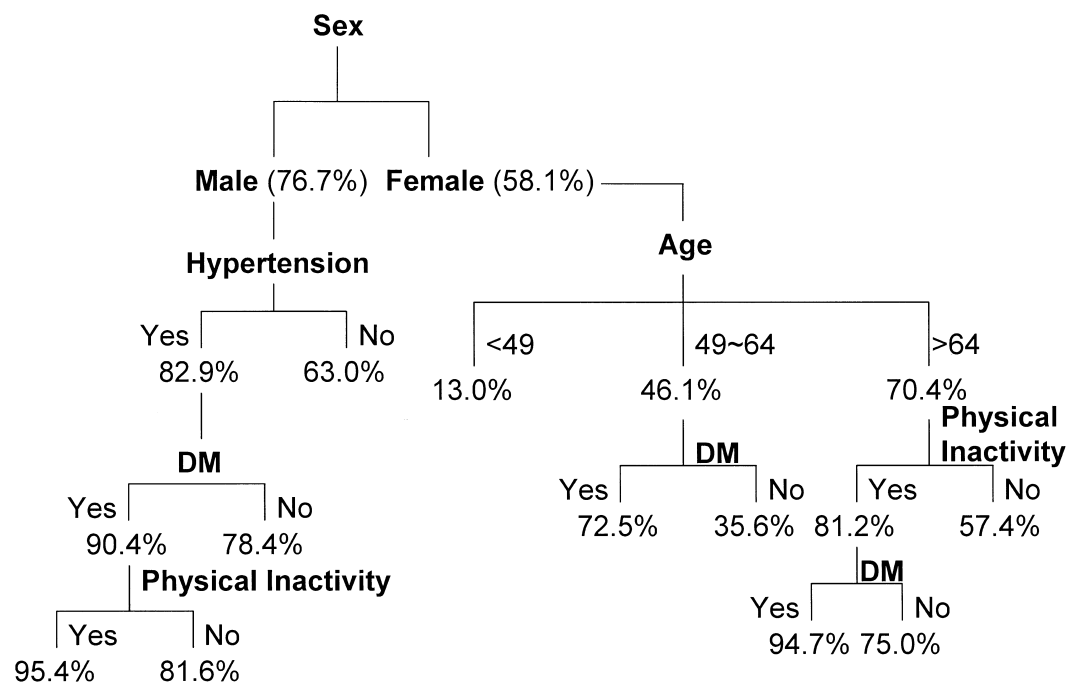

Figure. Prediction model results for coronary angiography by CHAID. 
Table III. Summarization of Subgroups Obtained from Exhaustive CHAID

\begin{tabular}{|c|c|c|}
\hline Subgroup & Risk factors & Probability $(\%)$ \\
\hline 1 & $\begin{array}{l}\text { Male; (High normal, } 130 / 80<\mathrm{BP}<140 / 90 \text { or Mild hypertension, } 140 / 90<\mathrm{BP} \\
<159 / 99 \text { or Moderate hypertension, } 160 / 100<\mathrm{BP}<179 / 109 \text { or Severe hyper- } \\
\text { tension, BP }>180 / 110 \text { ); Diabetes; Physical inactivity. }\end{array}$ & 95.4 \\
\hline 2 & $\begin{array}{l}\text { Male, } \text { (High normal, } 130 / 80<\mathrm{BP}<140 / 90 \text { or Mild hypertension, } 140 / 90<\mathrm{BP} \\
<159 / 99 \text { or Moderate hypertension, } 160 / 100<\mathrm{BP}<179 / 109 \text { or Severe hyper- } \\
\text { tension, } \mathrm{BP}>180 / 110 \text { ); No physical inactivity; Diabetes. }\end{array}$ & 81.6 \\
\hline 3 & $\begin{array}{l}\text { Male, } \text { (High normal, } 130 / 80<\mathrm{BP}<140 / 90 \text { or Mild hypertension, } 140 / 90<\mathrm{BP} \\
<159 / 99 \text { or Moderate hypertension, } 160 / 100<\mathrm{BP}<179 / 109 \text { or Severe hyper- } \\
\text { tension, } \mathrm{BP}>180 / 110 \text { ); No diabetes. }\end{array}$ & 78.4 \\
\hline 4 & Male; no hypertension, blood pressure (BP) normal. & 63.0 \\
\hline 5 & Female; age $\leqq 49$ & 13.0 \\
\hline 6 & Female; age $49-64$; Diabetes. & 72.5 \\
\hline 7 & Female; age 49-64; No diabetes. & 35.6 \\
\hline 8 & Female; age >64; Physical inactivity; Diabetes. & 94.7 \\
\hline 9 & Female; age $>64$, Physical inactivity. & 75.0 \\
\hline 10 & Female; age $>64$, No physical inactivity. & 57.4 \\
\hline
\end{tabular}

Table IV. Misclassification Matrix

\begin{tabular}{lcccr}
\hline & & Actual category & \\
& & Positive & Negative & Total \\
\hline \multirow{3}{*}{ Predicted category } & Positive & 1280 & 394 & 1674 \\
& Negative & 112 & 216 & 328 \\
& Total & 1392 & 610 & 2002 \\
\hline
\end{tabular}

the following age groups: under 49: $13.0 \%$, between 49 and 64: $46.1 \%$, and older than 64: 70.4\%. In further segmentation for females aged between 49 and 64, those with diabetes had a $72.5 \%$ chance compared with $35.6 \%$ for those without. Physical inactivity produced a further variable for females over 64 years old; $81.2 \%$ for physically inactive patients and $57.4 \%$ for physically active ones. Finally, the highest risk female group $(94.7 \%)$ consisted of those who were above 64 , physically inactive, and suffering from diabetes. Without diabetes the probability dropped to $75.0 \%$.

Ten homogeneous groups were obtained, with the probabilities of developing significant CHD ranging from as high as $95.4 \%$ to as low as $13.0 \%$. Table III summarizes the risk factors and probabilities for each group.

Sensitivity, specificity, and true positive predictive value: The misclassification matrix model is shown in Table IV. The sensitivity, specificity, and predictive accuracy of the exhaustive CHAID model are $92.0 \%, 35.4 \%$, and $76.5 \%$, respectively. 


\section{DISCUSSION}

Coronary angiography is both a highly utilized technique and an invasive diagnostic procedure, making the presence of a high false positive rate enormously expensive as well as potentially disastrous. Among our samples, 30.5\% received an insignificant result (normal or near-normal). A previous study reported a rate of 20 to $24 \%$. $^{3,4)}$ This clearly demonstrates the necessity of establishing an evidence-based prediction model. Our study found a significant variation across homogeneous groups in a large regional analysis of patients with suspected CHD, indicating that the model established in this study is a useful prediction model for prescribing coronary angiography. Some important findings are as follows.

1. As previously reported, the probability of developing significant CHD varied according to risk factors such as sex, hypertension, diabetes, age, and level of physical activity. Our study, however, goes further in its examination of the influence of interactions among patient characteristics on the outcome of coronary angiography. For example, MacMahon, et al $l^{9)}$ established that hypertension could be a predictor variable. This is confirmed by the present study but qualified with the addition that hypertension only has an impact on the probability of developing significant CHD for males. As opposed to hypertension, age only affects the probability of developing significant CHD for females. In addition, Barrett-Connor ${ }^{11)}$ found that patients with diabetes had a greater likelihood of developing CHD than patients without diabetes. Again, our research concurs, but adds that this probability differs according to its interaction with sex, level of physical activity, and age. This is demonstrated clearly by, for example, males with severe hypertension and diabetes having a $90.4 \%$ chance of developing significant CHD, physically inactive females with diabetes having a $94.7 \%$ chance, and middle-aged females suffering from diabetes having only a $72.5 \%$ chance.

2. By taking into account the interactions among variables, our study produced 10 homogeneous groups from the analysis of CHAID, with the probabilities for each group ranging from $13.0 \%$ to $95.4 \%$. Although all suspected patients have positive results from treadmill exercise testing or nuclear myocardial perfusion scanning, the actual probability of developing significant CHD differs dramatically according to the predictor variables.

3. Our study reveals numerous interactions among categorical data. For example, age only has an impact on the probability of developing significant CHD for suspected female patients, in contrast to hypertension which only has an impact in males. Although physical inactivity has an impact in both males and females, the degree differs depending on the specific variables. For example, diabetes increases the probability of developing significant CHD from 78.4 to $90.4 \%$ 
for male patients with hypertension, while it increases the probability from 75.0 to $94.7 \%$ for physically inactive females. This propensity for interactions between variables has only been highlighted by one previous study. ${ }^{21)}$

4. The sensitivity, specificity, and positive predictive value of our study are $92.0 \%, 35.4 \%$, and $76.5 \%$, respectively. The sensitivity and specificity of the exercise treadmill test in the diagnosis of CHD have been reported to be $58 \%$ to $82 \%$ and $57 \%$ to $95 \%$, respectively. ${ }^{22-25)}$ The high sensitivity rate is strong evidence that the diagnostic accuracy of our study is at least as high as the treadmill exercise test. However, the low specificity rate is due to over $90 \%$ of our study population yielding a positive finding in the treadmill exercise ECG test or nuclear myocardial perfusion scanning.

5. A previous study has shown that premenopausal females younger than 49 have a significantly reduced chance of developing significant CHD in comparison with those older than $49 .{ }^{26)}$ The probability obtained in the present study was $13.0 \%$, confirming this finding.

6. Schnohr, et al found that smoking was a significant risk factor, ${ }^{27)}$ however, our study has not confirmed this finding. The most probable reason for this is that only one independent variable can show a significant result. The previous study was done in Denmark, while the present study was conducted in Taiwan, where only a fraction of the smoking population is female. Therefore the significant result may have originated in gender rather than smoking.

7. To compare CHAID with common statistical techniques, we conducted logistic regression using the same dataset with the angiography result being the dependent variable and others being independent variables. Both models produced similar results. Except for a family history of cardiovascular disease and smoking, which are statistically significant only in the logistic regression model, gender, age, hypertension, diabetes, and physical inactivity are statistically significant predictors in both models. However, in contrast to logistic regression, CHAID demonstrates the interactions among independent variables. For example, the results of CHAID from this study show that hypertension is a statistically significant predictor for male patients, but not statistically significant for female patients. In terms of the accuracy of prediction, the CHAID result is more concordant with data than that of logistic regression. For example, among 76 older female (aged more than 64 years) patients with diabetes and a low level of physical activity, $72(94.7 \%)$ were diagnosed as having CHD. A forecasting model developed from forward stepwise logistic regression shows only an $80 \%$ probability of having CHD.

Implications: The findings of this study have important implications for the established prediction model that directs the decisions concerning the use of coronary angiography as well as prioritizing the patients who will undergo the pro- 
cedure. Previous studies have revealed that waiting time is associated with the outcome. ${ }^{28,29)}$ Sampalis, et al found that immediately before coronary artery bypass surgery, those who had waited longer than 97 days had significantly reduced physical functioning compared with patients who had waited 97 days or less. ${ }^{30)}$ Assuming the inevitability of a period of waiting, our study can assist health care workers to prioritize suspected CHD patients who need coronary angiography, in other words, patients with a high risk having priority over those at a low risk.

Although the model established in the current study has good sensitivity, specificity, and positive predictive values, we recommend against physicians using them to replace the treadmill exercise test or nuclear myocardial perfusion scanning without further cross-validation. It must be remembered that high normal and near-normal rates of treadmill exercise testing increase significantly the cost of coronary angiography. The evidence-based tree diagram in this current study can also assist physicians to enhance the accuracy of a diagnosis to reduce expenses.

CHAID has been proven to be an effective analytic technique for eliminating interactions among categorical variables, although it has not been adopted in previous studies of the risk factors of coronary artery disease. Our research is consequently a pioneering study utilizing this method to establish a prediction model for coronary angiography. The present study has demonstrated its validity for use on large datasets by discovering numerous interactions missed by previous studies.

Disease management has been successfully advocated to reduce health care expenses in several countries within the OECD. The key to success in disease management is the identification and targeting of high utilization groups according to risk factors. Our tree diagram can provide the necessary information needed for such a process. In addition, the current evidence-based model could provide managers and policy-makers with a serious advantage in their efforts to reduce unnecessary care.

As mentioned above, females younger than 49 have only a $13.0 \%$ chance of developing significant CHD. This has significant implications for a physician's risk assessments prior to coronary angiography. When assessing a female younger than 49, clinicians should only prescribe coronary angiography once they have established the presence of additional, higher risk factors.

Limitations: In interpreting our findings, two limitations must be acknowledged. First, although we included all the variables mentioned in previous studies into our research, our findings may still be affected by not including universal variables; future studies could, therefore, expand on the variables taken into account. Second, all cases in this study are collected from the claim dataset of the National 
Health Insurance Program in Taiwan. Studies have revealed that risk factors of CHD differ from country to country; therefore, it is not clear how our findings can be generalized to patients with varying risk factors. Future research could work towards cross-validating this model among different countries.

\section{REFERENCES}

1. Finesilver C. Reducing stress in patients having cardiac catheterization. Am J Nurs 1980; 80: 1805-7.

2. Davis K, Kennedy JW, Kemp HG Jr, Judkins MP, Gosselin AJ, Killip T. Complications of coronary arteriography from the Collaborative Study of Coronary Artery Surgery (CASS). Circulation 1979; 59: 1105-12.

3. Chaikhouni A, Gehani AA, Horak J, Hajar HA. Coronary angiography in Qatar: the first ten years. Angiology 1998; 49: 625-30.

4. Kirchgatterer A, Weber T, Auer J, et al. Analysis of referral diagnoses of patients with normal coronary angiogram. Wien Klin Wochenschr 1999; 111: 434-8. (German)

5. Kannel WB, Larson M. Long-term epidemiologic prediction of coronary disease. The Framingham experience. Cardiology 1993; 82: 137-52.

6. Lerner DJ, Kannel WB. Patterns of coronary heart disease morbidity and mortality in the sexes: a 26-year follow-up of the Framingham population. Am Heart J 1986; 111: 383-90.

7. Wannamethee SG, Shaper AG, Walker M. Physical activity and mortality in older men with diagnosed coronary heart disease. Circulation 2000; 102: 1358-63.

8. Anderson KM, Wilson PW, Odell PM, Kannel WB. An updated coronary risk profile. A statement for health professionals. Circulation 1991; 83: 356-62.

9. MacMahon S, Peto R, Cutler J, et al. Blood pressure, stroke, and coronary heart disease. Part 1, Prolonged differences in blood pressure: prospective observational studies corrected for the regression dilution bias. Lancet 1990; 335: 765-74.

10. Black HR. Metabolic considerations in the choice of therapy for the patient with hypertension. Am Heart $\mathrm{J}$ 1991; 121: 707-15. (Review)

11. Barrett-Connor EL, Cohn BA, Wingard DL, Edelstein SL. Why is diabetes mellitus a stronger risk factor for fatal ischemic heart disease in women than in men? The Rancho Bernardo Study. JAMA 1991; 265: 627-31.

12. Leon AS, Connett J. Physical activity and 10.5 year mortality in the Multiple Risk Factor Intervention Trial (MRFIT). Int J Epidemiol 1991; 20: 690-7.

13. Kaprio J, Norio R, Pesonen E, Sarna S. Intimal thickening of the coronary arteries in infants in relation to family history of coronary artery disease. Circulation 1993; 87: 1960-8.

14. Castelli WP. Epidemiology of triglycerides: a view from Framingham. Am J Cardiol 1992; 70: 3H-9.

15. Collins R, Peto R, MacMahon S, et al. Blood pressure, stroke, and coronary heart disease. Part 2. Short-term reductions in blood pressure: overview of randomised drug trials in their epidemiological context. Lancet 1990; 335: 827-38. (Review)

16. Brezinka V, Padmos I. Coronary heart disease risk factors in women. Eur Heart J 1994; 15: 1571-84. (Review)

17. Kass GV. An exploratory technique for investigating large quantities of categorical data. J Applied Statistics 1980; 29: 119-27.

18. Biggs D, de Ville B, Suen E. A method of choosing multiway partitions for classification and decision trees. J Applied Statistics 1991; 18: 49-62.

19. Huang HC, Lin TK, Ngui PW. Analyzing a mental health survey by chi-squared automatic interaction detection. Annals of The Academy of Medicine, Singapore 1993; 22: 332-7.

20. Levi L, Guilburd JN, Bar-Yosef G, Zaaroor M, Soustiel JF, Feinsod M. Severe head injury in children-analyzing the better outcome over a decade and the role of major improvements in intensive care. Childs Nerv Sys 1998; 14: 195-202.

21. Kannel WB, Higgins M. Smoking and hypertension as predictors of cardiovascular risk in population studies. J Hypertens 1990; 8 (Suppl): S3-8. (Review) 
22. McNeer JF, Margolis JR, Lee KL, et al. The role of the exercise test in the evaluation of patients for ischemic heart disease. Circulation 1978; 57: 64-70.

23. Bartel AG, Behar VS, Peter RH, Orgain ES, Kong Y. Graded exercise stress tests in angiographically documented coronary artery disease. Circulation 1974; 49: 348-56.

24. Goldschlager N, Selzer A, Cohn K. Treadmill stress tests as indicators of presence and severity of coronary artery disease. Ann Intern Med 1976; 85: 277-86.

25. Gianrossi R, Detrano R, Mulvihill D, et al. Exercise-induced ST depression in the diagnosis of coronary artery disease. A meta-analysis. Circulation 1989; 80: 87-98. (Review)

26. Gurevitz O, Jonas M, Boyko V, Rabinowitz B, Reicher-Reiss H. Clinical profile and long-term prognosis of women $<$ or $=50$ years of age referred for coronary angiography for evaluation of chest pain. Am J Cardiol 2000; 85: 806-9.

27. Schnohr P, Jensen JS, Scharling H, Nordestgaard BG Coronary heart disease risk factors ranked by importance for the individual and community. A 21 year follow-up of 12000 men and women from The Copenhagen City Heart Study. Eur Heart J 2002; 23: 620-6.

28. Meier-Kriesche H, Port FK, Ojo AO, et al. Deleterious effect of waiting time on renal transplant outcome. Transplant Proc 2001; 33: 1204-6.

29. Mayo NE, Scott SC, Shen N, Hanley J, Goldberg MS, MacDonald N. Waiting time for breast cancer surgery in Quebec. CMAJ 2001; 164: 1133-8.

30. Sampalis J, Boukas S, Liberman M, Reid T, Dupuis G. Impact of waiting time on the quality of life of patients awaiting coronary artery bypass grafting. CMAJ 2001; 165: 429-33. 